\title{
Mobilização precoce na fase aguda da trombose venosa profunda de membros inferiores
}

\author{
Early mobilization in acute stage of deep venous thrombosis of the \\ lower limbs
}

\author{
Geane de Souza Penha ${ }^{1}$, Ana Paula Damiano², Tales de Carvalho³ ${ }^{3}$ Vinícius Lain ${ }^{4}$, João Daniel Serafim ${ }^{4}$
}

\section{Resumo}

O tratamento convencional da trombose venosa profunda na fase aguda consiste em restrição ao leito. Porém, estudos recentes contestam essa abordagem terapêutica, enfatizando que a mobilização precoce propicia resultados clínicos favoráveis. O objetivo deste estudo foi pesquisar em literatura científica, principalmente ensaios clínicos controlados, sobre a mobilização precoce de pacientes portadores de trombose venosa profunda de membros inferiores na fase aguda. Utilizou-se como estratégia de pesquisa o site PubMed para a busca de estudos relacionados à mobilização precoce, deambulação e trombose venosa profunda na fase aguda. Os artigos consultados abrangeram o período de 1992 a 2007. Em todos os estudos, a mobilização precoce esteve associada à heparina de baixo peso molecular e a terapia de compressão. Estudos avaliados nesta revisão têm demonstrado os benefícios na redução da dor e edema, com melhora da qualidade de vida, pela estratégia terapêutica de mobilização precoce em combinação com anticoagulação e compressão da perna na trombose venosa profunda, sem que ocorra maior risco de desfechos relevantes, como embolia pulmonar e morte.

Palavras-chave: Trombose venosa, deambulação, compressão, extremidade inferior.

\section{Introdução}

A trombose venosa profunda (TVP) é uma doença que se caracteriza pela formação aguda de trombos que acometem as veias profundas dos membros, acarretando obstrução parcial ou total ${ }^{1-3}$.

Segundo Labas et al. ${ }^{4}$, a TVPé uma doença vascular comum e potencialmente ameaçadora à vida. No Brasil, $\mathrm{Maffei}^{5}$ relata incidência de 0,6 casos por 1.000 habitantes/ano, a partir dos casos de TVP confirmados

\begin{abstract}
Conventional treatment of deep venous thrombosis in the acute phase is bed confinement. However, recent studies have challenged such therapeutic approach, emphasizing that early mobilization provides favorable clinical outcomes. This study aimed at finding qualified scientific studies, especially controlled clinical trials, on early mobilization of patients with acute deep venous thrombosis of the lower limbs. PubMed was used to search for articles related to early mobilization, ambulation and acute deep venous thrombosis. Articles covered the period from 1992 to 2007. In all studies, early mobilization was associated with low molecular weight heparin and compression therapy. Studies evaluated in this review showed benefits in reducing pain and edema, with improvement in quality of life, using the therapeutic strategy of early mobilization in combination with anticoagulation and compression of the leg in patients with deep venous thrombosis, without increased risk of relevant outcomes, such as pulmonary embolism and death.
\end{abstract}

Keywords: Venous thrombosis, ambulation, compression, lower extremity.

por flebografia ou duplex scan. Em trabalho de revisão sistemática (metanálise), Fowkes et al. ${ }^{6}$, em 2003, estimaram incidência mundial de TVP de 0,5 casos por 1.000 habitantes/ano. Bastante comum em hospitais, a TVP acomete 84 pessoas por 100.000 habitantes/ano ${ }^{7}$, sendo a causa mais comum de morbidade e mortalidade em pacientes cirúrgicos. É responsável por 300.000

1. Acadêmica de Fisioterapia, Universidade do Estado de Santa Catarina (UDESC), Florianópolis, SC.

2. Mestranda, UDESC, Florianópolis, SC.

3. Doutor. Médico especialista, Cardiologia e Medicina do Esporte. Coordenador, professor, Núcleo de Cardiologia e Medicina do Exercício, Centro de Ciências da Saúde e do Esporte (CEFID), UDESC, Florianópolis, SC.

4. Especialista, Cirurgia Vascular, Hospital Regional de São José Dr. Homero de Miranda Gomes, São José, SC.

Não foram declarados conflitos de interesse associados à publicação deste artigo.

Artigo submetido em 02.08.08, aceito em 07.11.08. 
a 600.000 hospitalizações a cada ano ${ }^{8}$. A TVP está presente em 20 a $35 \%$ dos óbitos intra-hospitalares e é associada à embolia pulmonar (EP) em 10 a 20\% dos casos, em estudos baseados em necropsias ${ }^{9-11}$.

As complicações provenientes da TVP repercutem negativamente em termos socioeconômicos e na qualidade de vida, sendo causa de morte precoce do indivíduo acometido. A EP é uma das manifestações mais temidas, sendo a terceira causa de morte por doenças cardiovasculares $^{1,2}$. Outra complicação não-mortal dessa afecção, porém de grande repercussão para a realização de atividades da vida diária, é a insuficiência venosa crônica, nesse caso, denominada síndrome póstrombótica, decorrente de dano progressivo e permanente do sistema valvular venoso durante o lento processo de lise dos coágulos. Tal síndrome causa sequelas graves, como varizes e úlcera venosa, o que deixa evidente a importância de uma intervenção terapêutica precoce e adequada que reduza a possibilidade de complicações graves e óbito ${ }^{1}$. A mobilização precoce tem sido considerada procedimento contraindicado durante a fase aguda de TVP, pela crença de que a contração muscular provocaria desprendimento do trombo da parede vascular, ocasionando $\mathrm{EP}^{1-3}$.

Em contrapartida, estudos recentes têm sugerido que a mobilização precoce não aumentaria o risco de EP, mas propiciaria benefícios na fase aguda da $\mathrm{TVP}^{12-18}$. Diante da controvérsia exposta, o objetivo deste estudo foi pesquisar estudos científicos qualificados, principalmente ensaios clínicos controlados, sobre a mobilização precoce de pacientes portadores de TVP de membros inferiores na fase aguda.

\section{Fatores de risco}

A TVP é considerada uma doença multifatorial ${ }^{1}$. A imobilidade no leito e as varizes são fatores de risco dos mais importantes, da estase venosa ${ }^{19}$. Os fatores trombogênicos podem estar presentes em neoplasias, haja vista que tumores podem aumentar a ação dos fatores pró-coagulantes ${ }^{3}$. A idade avançada também é considerada fator de risco para a TVP, porque no envelhecimento ocorre diminuição da atividade fibrinolítica, elevação da resistência vascular e dilatação venosa, com consequente redução da velocidade do fluxo sanguíneo ${ }^{1,2}$. Existe maior incidência no sexo feminino, que é frequentemente relacionada ao uso de medicação anticoncepcional, tendo em vista que os estrógenos aumentam os níveis sanguíneos de fatores de coagulação ${ }^{20}$. A obesidade é um fator de risco independente para o desenvolvimento da TVP, uma vez que inibe a atividade fiblinolítica e dificulta a deambulação ${ }^{1,5}$.

$\mathrm{Na}$ cirurgia de prótese total do quadril, a abordagem ântero-lateral proporciona incidência maior de $\mathrm{TVP}^{21}$, devido à torção da veia femoral profunda, durante as manobras de adução e rotação externa forçadas ${ }^{22}$.

\section{Etiopatogenia}

A etiopatogenia da TVP é explicada pela clássica tríade de Virchow: estase venosa, lesão endotelial e hipercoagulabilidade ${ }^{1-3,5}$. A estase venosa é vista como o principal fator predisponente da TVP. A diminuição do fluxo sanguíneo leva ao aumento da quantidade de sangue nas veias, dilatando-as passivamente, com consequente redução da velocidade do fluxo sanguíneo. Desse modo, tais alterações decorrem de: 1) queda no débito cardíaco, relaxamento muscular durante o repouso, anestesia e paralisias; 2) déficit da bomba venosa periférica. Portanto, a diminuição da velocidade do fluxo sanguíneo nas veias ocasiona a perturbação do fluxo laminar, causando acúmulo local de hemácias, plaquetas e leucócitos. Esse depósito celular é estabilizado pela constituição de uma rede de fibrina que prende tais elementos, culminando com a formação do trombo ${ }^{2,5}$.

O endotélio normal é uma superfície nãotrombogênica sobre a qual não aderem plaquetas, nem ocorre ativação de proteínas coagulantes. Quando, porém, existe lesão endotelial, ocorre uma exposição do subendotélio, favorecendo a agregação de plaquetas e glóbulos brancos, o que desencadeia a ativação dos mecanismos de coagulação, com formação do trombo ${ }^{23}$.

A hipercoagulabilidade está presente quando há aumento de fatores de coagulação e redução dos fatores inibidores da coagulação, o que pode ocorrer em determinados estados fisiológicos, patológicos e terapêuticos, como na gravidez, câncer, trombofilia e uso de medicamentos esteroides e quimioterápicos ${ }^{24}$. 


\section{Diagnóstico clínico}

A grande maioria das TVP tem início insidioso, com poucas manifestações clínicas ou apresenta-se em caráter assintomático. São sintomas e sinais clínicos clássicos da TVP: dor a palpação muscular, dor espontânea, empastamento da panturrilha, edema subcutâneo e muscular, distensão venosa superficial e aumento da temperatura do membro afetado. As alterações da coloração da pele são mais comuns nas TVP proximais severas, com coloração cianótica pela obstrução do segmento (phlegmasia cerulea) ou palidez pela presença do vasoespasmo (phlegmasia alba) $)^{1-3}$.

\section{Métodos diagnósticos}

A ultrassonografia tem sido o método de escolha para diagnóstico de TVP proximal, por não ser invasivo e apresentar boa sensibilidade e especificidade ${ }^{5,24}$. Na avaliação pela ultrassonografia, o Doppler incorporado ao exame permite a detecção da menor influência dos movimentos ventilatórios pulmonares na modulação da velocidade do fluxo sanguíneo, o que contribui para o diagnóstico da TVP. A avaliação pela ultrassonografia oferece maior sensibilidade e especificidade quando se trata do segmento fêmoro-poplíteo ou ilíaco-femoral ${ }^{5}$.

Entretanto, a flebografia, um procedimento invasivo, é considerado como o padrão-ouro para o diagnóstico da TVP. Permite uma visualização global do sistema venoso, por meio da aplicação de contraste iodado em veias do pé. Pela falha no enchimento venoso em determinado segmento se constata a presença do trombo ${ }^{3,5}$. A flebografia radioisotópica é um método de valor histórico empregado em alguns estudos científicos, consistindo da injeção de microesferas de albumina marcadas com tecnécio-99m, cujos trajetos nas veias são visualizados pela cintilografia. O diagnóstico do trombo é feito pela ausência de visualização do vaso e, mais tardiamente, pela presença de concentração de substância radiativa no local de um trombo. É possível empregá-la em pacientes com suspeita de EP, uma vez que pode ser realizada simultaneamente a cintilografia pulmonar ${ }^{24}$.

\section{Tratamento medicamentoso}

O tratamento hospitalar da TVP pode ser realizado com heparina não-fracionada por via intravenosa e subcutânea. Atualmente, porém, vem sendo dada preferência, no tratamento da TVP, à heparina de baixo peso molecular, utilizando a via subcutânea, de modo que pode ser utilizada no tratamento ambulatorial das tromboses venosas não-complicadas, por apresentar menor potencial hemorrágico em comparação com a heparina não-fracionada. A heparina de baixo peso molecular difere da heparina não-fracionada por apresentar maior atividade antiXa, maior biodisponibilidade com dosagens menores, maior meia vida e maior preditibilidade na resposta anticoagulante quando administrada em dosagens fixas, não exigindo rigoroso controle laboratorial, como as demais ${ }^{25}$. Após a fase aguda, o paciente deve ser mantido anticoagulado por meio de terapia oral $^{4,25}$.

\section{Compressão venosa}

A terapia de compressão é o tratamento sintomático da TVP, com aplicação de uma pressão exercida e transmitida aos tecidos, com a finalidade de aumentar a pressão intravenosa, orientar o fluxo venoso de retorno e potencializar a fração de ejeção da panturrilha, de modo que ocorra a resolução do edema ${ }^{17,26}$. As ataduras são tiras de tecido elástico ou inelástico. Quanto menor o grau de elasticidade de uma atadura, mais profundamente ela atuará. A bota de Unna é uma compressão inelástica composta de ataduras de crepe embebida em pasta com gelatina incolor, glicerina, óxido de zinco e água, normalmente usada no tratamento para úlcera venosa. As meias de compressão elástica são adicionadas no tratamento da TVP para prevenir a síndrome póstrombótica ${ }^{26}$. A compressão máxima é exercida no tornozelo e vai decrescendo em direção à coxa ${ }^{27}$.

\section{Métodos}

Utilizou-se como estratégia a pesquisa em indexadores, como PubMed, LILACS e SciELO, para a busca de artigos relacionados à mobilização precoce, deambulação e TVP na fase aguda. Foram selecionadas 15 referências, todas publicadas na língua inglesa. Os artigos consultados abrangeram o período entre 1992 e 2007.

\section{Resultados}

No passado, o repouso prolongado no leito era considerado essencial para o tratamento da TVP com intuito de controlar a propagação trombótica e reduzir o risco de $\mathrm{EP}^{14}$. Por outro lado, o descanso no leito por um período de 7 a 10 dias é ainda parte do tratamento da TVP 
em muitos centros hospitalares ${ }^{12}$. No entanto, os resultados de algumas experiências controladas sugerem que a mobilização precoce é uma modalidade terapêutica segura e eficaz para tratar aspectos clínicos da TVP $^{12-15,17,18}$. Partsch \& Blättler ${ }^{16}$ relataram estudo experimental controlado com a finalidade de avaliar os benefícios da compressão e exercícios de caminhada em comparação com repouso no leito na fase aguda da TVP proximal, diagnosticada por eco-Doppler ou flebografia. A amostra foi composta por 45 pacientes heparinizados, divididos em três grupos de 15 pacientes, seguindo distintas modalidades terapêuticas durante 10 dias. Os indivíduos do grupo A receberam bandagem de compressão inelástica (botas de Unna) sobre a perna e bandagem adesiva sobre a coxa, enquanto os do grupo B utilizaram meias elásticas compressivas classe II (compressão no tornozelo de 23 a $32 \mathrm{mmHg})^{26}$ até a extensão da coxa. Os pacientes dos dois primeiros grupos foram submetidos a programa de caminhadas. Já os indivíduos do grupo $\mathrm{C}$ foram mantidos ao repouso e não foram submetidos ao tratamento de compressão. Pelo podômetro, foi constatado que a distância caminhada nos grupos A e B variou entre 600 e $1.200 \mathrm{~m}$, enquanto no grupo de $\mathrm{C}$ a distância média de caminhadas diárias foi somente de $66 \mathrm{~m}$. O nível da dor foi estimado diariamente por meio da escala analógica visual (VAS - Visual Analogue Scale), que é a aferição subjetiva da intensidade da dor por meio de variação numérica de 0 a $10^{17}$, e do teste de Lowenberg, através do qual aplica-se pressão sobre a musculatura com o uso do manguito e registra-se o nível de pressão que o paciente refere dor $^{17}$. Nos grupos A e B, o grau de dor sofreu um decréscimo estaticamente significativo $(p<0,05)$ no segundo dia e, no grupo de repouso, apenas depois de 10 dias. No último dia de estudo, a redução do edema (avaliado com fita métrica) e a melhoria na pontuação clínica (avaliada por meio de escore clínico contendo sete itens: dor durante a caminhada; dor na planta do pé na posição ortostática; palpação dolorosa na panturrilha; edema subfascial e prefascial; hiperemia; cianose; e aumento da temperatura) foi significativamente mais pronunciada $(p<0,01)$ nos grupos da compressão em relação ao grupo de repouso. Não houve diferença significativa quanto à ocorrência de novos eventos de EP e progressão do trombo avaliados no primeiro e segundo dias de estudo, com cintilografia e eco-Doppler, respectivamente. Ademais, nenhuma complicação proveniente das terapias empregadas foi detectada. É interessante salientar que a comparação entre os grupos de compressão revelou que a estratégia aplicada aos pacientes do grupo A produziu efeitos superiores em reduzir os sinais e sintomas clínicos agudos. O estudo de Partsch \& Blättler ${ }^{16}$ sugeriu que pacientes com TVP proximal deveriam ser incentivados a caminhar com bandagens ou meias de compressão, visando a uma diminuição importante de sinais e sintomas clínicos agudos, sem aumentar o risco de EP.

Blättler \& Partsch ${ }^{27}$ realizaram um estudo prospectivo randomizado com a finalidade de verificar se a utilização de heparina, associada a compressão e a caminhada, proporcionaria melhor evolução clínica do que o repouso no leito. A amostra consistiu de 53 pacientes com TVP proximal sintomática, todos heparinizados, divididos em três grupos submetidos a distintas terapias. Nos pacientes do grupo A $(n=18)$ foi aplicada bandagem de compressão inelástica, e nos pacientes do grupo B ( $\mathrm{n}=18)$, meias de compressão elástica. Os pacientes de ambos os grupos foram encaminhados imediatamente para a deambulação. Os pacientes do grupo $\mathrm{C}(\mathrm{n}=17)$ permaneceram em repouso absoluto, sem que fossem submetidos a terapêutica compressiva. A varredura pulmonar e eco-Doppler da perna foram realizadas nos dias 0 e 9 . Nos grupos em que foi utilizada a terapêutica compressiva, a distância caminhada diariamente, determinada por meio de podômetro, aumentou no decorrer do tempo, atingindo $4 \mathrm{~km} /$ dia em média. A melhoria do bem-estar geral (foi avaliada com uso da VAS) e a qualidade de vida (questionário de qualidade de vida específico para doença venosa contendo 10 itens) foram significativamente superiores nos grupos de compressão $(\mathrm{p}<0,05$ para meia e $\mathrm{p}<0,001$ para bandagem $)$ em comparação com o grupo de repouso. O repouso significou pouca influência sobre o edema nas pernas, ao passo que a terapia de compressão associada a mobilização precoce conduziu a uma rápida e drástica redução da circunferência da perna $(\mathrm{p}<0,001)$. Na pontuação clínica, os grupos de compressão obtiveram resultados significativamente melhores do que o grupo de repouso $(\mathrm{p}<0,001)$. A dor avaliada diariamente através da VAS indicou que houve uma diminuição gradativa de dor em todos os grupos $(\mathrm{p}<0,001)$; porém, 
ocorreu melhora mais rápida durante os primeiros 4 dias nos grupos de compressão $(\mathrm{p}<0,01)$. O melhor efeito foi alcançado no grupo de bandagem, sendo observada quase ausência de dor no final do período de observação $(\mathrm{p}<0,01)$. A dor induzida pelo teste de Lowenberg revelou que no grupo de repouso a dor reduziu consideravelmente em 3 dias, mas houve recorrência, com a dor sendo constante e acentuada ao longo dos 6 dias subsequentes. Porém, nos grupos de deambulação precoce ocorreu diminuição da dor de forma progressiva desde o início da terapia, sem recorrência. A progressão do trombo, verificada no primeiro e segundo dias de estudo com eco-Doppler, foi menos frequente e menos pronunciada nos grupos A e B em relação ao grupo $\mathrm{C}(\mathrm{p}<$ 0,01). Não houve diferença de novos eventos de EP no segundo exame de varredura pulmonar entre os grupos. O estudo de Blättler \& Partsch ${ }^{27}$ sugeriu ser a compressão na perna combinada com a caminhada a melhor estratégia terapêutica para o tratamento de pacientes sintomáticos com TVP proximal, sendo superior à estratégia de repouso absoluto no leito.

Isma et al. ${ }^{14}$ realizaram estudo randomizado no Hospital Universitário de Malmo, Suécia, com o propósito de avaliar se o exercício precoce supervisionado melhoraria a recanalização e reduz os sintomas clínicos da TVP diagnosticada por meio de flebografia. Os 72 pacientes incluídos no estudo foram seguidos durante 6 meses, após serem divididos em: grupo experimental $(\mathrm{n}=36)$, com anticoagulação, meias de compressão classe II e exercício supervisionado; e grupo controle $(\mathrm{n}=36)$ recebendo a mesma terapia, exceto em relação ao exercício. Os pacientes foram incentivados a caminhar já na fase hospitalar. Entre 5 e 7 dias após o diagnóstico de TVP, todos os indivíduos no grupo experimental foram incentivados a se exercitar diariamente em casa por 15 minutos, durante 6 meses. Durante o primeiro mês, frequentavam sessão semanal de 45 minutos, supervisionada por fisioterapeuta. Nos meses subsequentes, frequentaram apenas 1 sessão mensal supervisionada. $\mathrm{O}$ programa de exercícios consistiu basicamente de caminhadas e exercícios resistidos, com ênfase nos membros inferiores. Foram avaliados: qualidade de vida (avaliada por meio de VAS); força muscular do quádriceps; equilíbrio; e circunferência da coxa e da panturrilha. Tais parâmetros foram mensurados em três diferentes ocasiões: à admissão ao estudo, durante o estudo e após 6 meses. Os resultados revelaram que não houve diferença entres os grupos. Para a avaliação da extensão e severidade da TVP na perna, foi utilizado o sistema de classificação de Bjorgell, que varia de 0 a $3(0=$ sem TVP, 1 = menos de um terço, $2=$ um terço ou mais, $3=$ dois terços ou mais da perna, referente ao segmento venoso comprometido), empregado durante a avaliação diagnóstica e depois de 6 meses, quando o protocolo do estudo foi finalizado. Os resultados indicaram que o grau de recanalização dos segmentos venosos afetados foi alto e não diferiu entre os grupos. Não houve TVP recorrente ou EP ou outras complicações do tratamento em nenhum indivíduo durante os 6 meses do período acompanhado, concluindo-se que a terapia proposta neste estudo não exacerbou agudamente o risco de complicações em pacientes com TVP. Nenhum beneficio da mobilização foi visto em relação ao grau de recanalização da trombose ou resolução rápida de dor ou edema e melhora na força muscular de quadríceps e equilíbrio. Todavia, este estudo demonstrou que o exercício precoce foi seguro em combinação com anticoagulação e meias de compressão, quando aplicados em pacientes com TVP.

No estudo randomizado de Aschwanden et al. ${ }^{13}$, o objetivo foi demonstrar que a mobilização precoce não aumentaria a frequência de EP em 129 pacientes com TVP proximal, diagnosticada por meio de eco-Dopller. Todos receberam terapia de heparina e foram submetidos à estrita imobilização (grupo A: $\mathrm{n}=60$ ) durante 4 dias ou a deambulação (grupo B: $n=69$ ) por 4 h/dia sob supervisão. Os indivíduos de ambos os grupos usaram meias de compressão classe II. Os pacientes foram investigados por meio de cintilografia ventilação /perfusão pulmonar quanto à presença de EP no início do estudo e no quarto dia. As alterações das circunferências das pernas e dor em repouso e durante o exercício foram avaliadas por meio de fita métrica e VAS, respectivamente. Os resultados encontrados demonstraram não haver diferença estatisticamente significativa entre os grupos, no que se refere à diminuição da circunferência e da dor em repouso nas pernas. No entanto, no grupo experimental foi observado decréscimo significativo na dor durante a prática de exercício. Todos os 
pacientes foram contatados em 3 meses e entrevistados a respeito de recorrência de TVP, sinais clínicos de EP, novas doenças concomitantes e ocorrência de maiores complicações. A incidência de EP no início do estudo foi 53 e 44,9\%, respectivamente nos indivíduos imóveis e móveis. Durante os 4 dias de observação ocorreram novos episódios de EP, com incidências de 10 e de 14,4\%, respectivamente, em pacientes do grupo A e B. Dos 16 casos de EP detectados no quarto dia, 12 ocorreram em indivíduos com EP prévia, correspondendo ao risco relativo 3,65, considerando-se a incidência de eventos tromboembólicos nos que já a haviam apresentado comparados com os que a apresentaram pela primeira vez. Não houve óbito durante os 4 dias de observação. No decorrer de 3 meses, a taxa de mortalidade foi 3,9\% ( 2 mortes no grupo A e 3 mortes no grupo B). As mortes foram ocasionadas por doenças malignas e houve três recorrências de TVP (1 do grupo A e 2 do grupo B). Os resultados deste estudo sugeriram ser a mobilização precoce recurso terapêutico efetivo e seguro para ser utilizada como rotina em pacientes com TVP, levando-se em consideração a comparação com a imobilização.

Partsch et al. ${ }^{15}$ desenvolveu estudo prospectivo no intuito de determinar a incidência de EP em 139 pacientes hospitalizados que apresentavam TVP proximal diagnosticada por flebografia radioisotópica, submetidos à terapia de anticoagulação, programa de caminhadas e compressão venosa. No início do estudo, 80 pacientes foram diagnosticados com EP, mas apenas 11 eram sintomáticos. No último dia de internação (11 dias em média), a cintilografia pulmonar realizada detectou novos casos de EP em quatro dos 59 sem histórico prévio e em sete com EP no primeiro exame. Porém, 33 pacientes $(23,7 \%)$ apresentaram regressão dos defeitos de perfusão na cintilografia. Um paciente de 80 anos de idade com carcinoma da próstata sofreu EP fatal. O estudo corroborou a informação de que existe uma relação direta entre TVP e EP, independente da mobilização precoce.

Partsch et al. ${ }^{16}$, em 1.289 pacientes com TVP sintomática, tratados com heparina de baixo peso molecular, compressão e exercícios de caminhada, determinou a incidência em curto prazo de EP, hemorragia, trombocitopenia induzida por heparina e morte. Os indivíduos foram avaliados por meio de cinco parâmetros durante o período de permanência hospitalar: presença de EP à admissão (V/Q scan) e incidência de EP após 10 dias (segunda V/Q scan), eventos fatais (necropsia), presença de doença maligna, complicações hemorrágicas e trombocitopenia induzida por heparina. A incidência de EP à admissão hospitalar foi: $53,4 \%$ no segmento ilíaco-femoral; 52,6\% na veia femoral; e 35,1\% nas veias da perna. Em dois terços dessas EP, os pacientes estavam assintomáticos. Nesse estudo, a baixa incidência de recidiva e EP fatal depõem a favor da deambulação precoce com compressão em membros inferiores em pacientes com sintomas de TVP.

Além dos já comentados neste presente artigo, existem muitos outros estudos que comprovaram a eficácia e a segurança de se considerar a mobilização precoce no contexto terapêutico da $\mathrm{TVP}^{4,12,18,28-32}$.

\section{Discussão}

Durante muito tempo, preconizou-se repouso absoluto no leito como tratamento da TVP aguda. O protocolo comumente utilizado na prática hospitalar consiste em repouso, elevação de membros inferiores e anticoagulantes até que se obtenha a estabilidade do trombo $^{1,15}$. Entretanto, muitos estudos sugerem ser a deambulação precoce recomendada para a maioria dos pacientes com TVP, devendo haver maior precaução com indivíduos com histórico de EP prévia ${ }^{13,15,17,18,29,30,32}$. Pesquisas têm demonstrado que a prescrição de repouso no leito para pacientes com TVP não reduziu a incidência de EP a ponto de influenciar significativamente a evolução clínica ${ }^{13,15,17,18,30,32}$. Do ponto de vista fisiopatológico, a imobilização produz consequências decorrentes da estase venosa, revelando-se como um dos mais importantes fatores discriminados por Virchow como sendo responsáveis pela trombogênese. A inatividade do mecanismo de bombeamento do sistema venoso proporcionado pelos músculos dos membros inferiores ("bomba muscular") e a atividade fibrinolítica deprimida são outros fatores que contribuem para a formação e propagação do trombo, com sequelas pós-trombóticas graves ${ }^{4,33}$.

Quando se compara a evolução de pacientes deixados em repouso no leito com os mobilizados precocemente, tem-se observado ausência de diferença 
significativa no aparecimento de $\mathrm{EP}^{13-17,28}$, embora se afirme que a mobilização precoce contribuiria para a redução da progressão do trombo ${ }^{16,28}$. Partsch et al. ${ }^{16}$ argumentou que a deambulação precoce poderia ser um aspecto protetor, uma vez que reduz a estase venosa, um dos fatores de risco para TVP recorrente ou progressiva. Os dados reportados por Partsch \& Blättler ${ }^{16}$ sugeriram que a deambulação em combinação com anticoagulação e compressão da perna em pacientes com TVP na fase aguda conduziria a uma regressão mais rápida de sintomas e sinais clínicos, como dor, edema, hiperemia e aumento de temperatura. Isma et al. ${ }^{14}$ corroborou, indicando que a mobilização precoce é o tratamento adequado para sintomatologia clínica dos pacientes, acrescentando ter sido observada melhora na qualidade de vida desses indivíduos.

Killewich et al. ${ }^{34}$ mencionam que regressão da TVP aguda ocorre devido ao aumento da atividade fibrinolítica endógena e do ativador de plasminogênio tecidual. O exercício físico acentua a atividade fibrinolítica endógena manifestada como uma diminuição no inibidor do ativador do plasminogênio ${ }^{34}$.

Fischer $^{35}$, em 1910, recomendava ataduras com emplastro de zinco para tratar pacientes com trombose, especulando que a firme compressão externa fixaria os coágulos na parede das veias. O efeito físico da compressão externa verificada no estudo realizado por Partsch et al. ${ }^{36}$, demonstrado por meio da flebografia, evidenciou ação contra a formação do edema decorrente do estreitamento das veias superficiais e profundas. Os mesmos autores concluíram que os materiais inelásticos, como o emplastro de zinco, são mais eficazes que o material elástico na redução da estase venosa local. Portanto, os resultados encontrados nos estudos realizados por Partsch \& Blättler ${ }^{16}$ e Blättler \& Partsch ${ }^{27}$ demonstraram que o grupo de compressão inelástica apresentou efeitos superiores em reduzir os sinais e sintomas clínicos. Em todos esses estudos mencionados, houve utilização de meias de compressão, o que impossibilitou avaliar o efeito isolado da mobilização precoce.

A propagação do trombo foi observada em cerca de $20 \%$ dos pacientes, apesar de tratamento adequado com heparina e mobilização tardia. Porém, esse valor cai para $1 \%$ se a mobilização for instituída precocemente ${ }^{37}$. A propagação do trombo foi relacionada ao nível de anticoagulação, tendo sido provavelmente influenciada pelo grau de estase ${ }^{37}$. Por isso, no paciente com TVP, tem sido proposta a mobilização aliada a heparina de baixo peso molecular imediatamente após o diagnóstico ${ }^{28,29,31,32,34}$.

No estudo realizado por Isma et al. ${ }^{14}$, não foi observado nenhum benefício substancial do exercício na recanalização venosa, haja vista que o acompanhamento foi tardio, efetuado 6 meses depois do fim do protocolo de estudo, inviabilizando a análise do efeito da mobilização na fase aguda. A pontuação de severidade relativamente baixa em ambos os grupos dificultou a avaliação dos eventuais efeitos do exercício.

Os sintomas provenientes da TVP resultam, parcialmente, de um aumento na pressão intracapilar e de posterior transudação de fluido dos capilares para o espaço intersticial ${ }^{38}$, ocasionados pelo obstáculo ao fluxo sanguíneo venoso e pela insuficiência valvar ${ }^{39}$. Tais fenômenos prejudicam a perfusão muscular da perna, promovendo a fadiga muscular ${ }^{38}$.

Os efeitos potencialmente benéficos da mobilização precoce relacionam-se à teoria da bomba muscular da panturrilha e ao treino muscular ${ }^{17}$. Durante a contração muscular, ocorre aumento na habilidade de ejeção, facilitando o retorno venoso, o que, por sua vez, reduz o gradiente de pressão hidrostática, responsável pela formação do edema ${ }^{40-42}$, bem como melhora a perfusão muscular potencializando sua ação ${ }^{38}$. O estudo de Partsch et al. ${ }^{16}$ corroborou ao relatar que compressão externa da perna, aliada ao programa de caminhadas, proporcionaria redução na pressão hidrostática sanguínea e, consequentemente, diminuiria os sintomas e sinais venosos.

A qualidade dos artigos, ensaios clínicos controlados e estudos epidemiológicos pesquisados neste estudo permitiu que os resultados, a discussão e, consequentemente, as conclusões, fossem fundamentadas em um bom grau de evidência científica. Os principais estudos pesquisados e citados são internacionais, oriundos de países desenvolvidos. Existe uma lacuna de pesquisas realizadas no Brasil e América Latina sobre o tema, 
diante da qual fica a sugestão para a realização de estudos experimentais no Brasil, o que poderia fornecer subsídios em prol da proposta de mobilização precoce no âmbito hospitalar em nosso meio.

\section{Conclusão}

Opondo-se à estratégia de restrição ao leito, estudos recentes, avaliados nesta revisão, demonstraram os benefícios na redução da dor e edema, com melhora da qualidade de vida, pela estratégia terapêutica de deambulação precoce em combinação com anticoagulação e compressão da perna em pacientes com TVP, sem que fosse verificada maior incidência de desfechos relevantes, como EP e morte.

\section{Referências}

1. Maffei FHA, Lastoria S, Yoshida WB, Rollo HA. Doenças vasculares periféricas. $3^{\text {a }}$ ed. Rio de Janeiro: Medsi; 2002. vol. 2.

2. Mello NA. Síndromes vasculares: clínica, diagnóstico, tratamento. São Paulo: Fundo Editorial BYK; 1999. p. 383.

3. Verstraete M, Vermylen J. Trombose. São Paulo: Sarvier; 1989. p. 356 .

4. Labas P, Ohrádka B, Vladimír J, Cambal M. The home treatment of deep vein thrombosis with low molecular weigth heparin, forced mobilisation and compression. Int Angiol. 2000;19:303-7.

5. Maffei, FHA. Trombose venosa profunda dos membros inferiores: incidência, patologia, fisiopatologia e diagnóstico. In: Maffei FHA, Lastoria S, Yoshida WB, Rollo HA. Doenças vasculares periféricas. $3^{\text {a }}$ ed. São Paulo: Medsi; 2002. p. $1363-86$.

6. Fowkes FJ, Price JF, Fowkes FG. Incidence of diagnosed deep vein thrombosis in the general population: systematic review. Eur J Vasc Endovasc Surg. 2003;25:1-5.

7. Anderson FA, Wheeler HB, Goldberg RJ, et al. A population based perspective of the hospital incidence and case-fatality rates of deep vein thrombosis and pulmonary embolism: the Worchester DVT Study. Arch Intern Med. 1991;151:933-8.

8. National Institutes of Health Consensus Conference. Prevention of venous thrombosis and pulmonary embolism. JAMA. 1986;256:744-9.

9. Diebold J, Löhrs U. Venous thrombosis and pulmonary embolism. A study of 5039 autopsies. Pathol Res Pract.1991;187:260-6.

10. Saeger W, Genzkow M. Venous thrombosis and pulmonary embolisms in post-mortem series: probable causes by correlations of clinical data and basic diseases. Pathol Res Pract. 1994;190:394-9.

11. Lindblad B, Sternby NH, Berqqvist D. Incidence of venous thromboembolism verified by necropsy over 30 years. BMJ. 1991;302:709-11.
12. Aldrich D, Hunt DP. When can the patient with deep venous thrombosis begin to ambulate? Phys Ther. 2004;84:268-73.

13. Aschwanden M, Labs KH, Engel H, et al. Acute deep vein thrombosis: early mobilization does not increase the frequency of pulmonary embolism. Thromb Haemost. 2001;85:42-6.

14. Isma N, Johanssson E, Björk A, et al. Does supervised exercise after deep venous thrombosis improve recanalization of occluded vein segments? A randomized study. J Thromb Thrombolysis. 2007;23:25-30.

15. Partsch H, Oburger K, Mostbeck A, König B, Köhn H. Frequency of pulmonary embolism in ambulant patients with pelvic vein thrombosis: a prospective study. J Vasc Surg. 1992; 16:715-22.

16. Partsch H, Blättler W. Compression and walking versus bed rest in the treatment of proximal deep venous thrombosis with low molecular weight heparin. J Vasc Surg. 2000;32:861-9.

17. Schellong SM, Schwarz T, Kropp J, Prescher Y, Beuthien-Baumann B, Daniel WG. Bed rest in deep venous thrombosis and the incidence of scintigraphic pulmonary embolism. Thromb Haemost. 1999;82(suppl 1):127-9.

18. Schaub RG, Simmons CA, Koets MH, Romano PJ 2nd, Stewart GJ. Early events in the formation of a venous thrombus following local trauma and stasis. Lab Invest. 1984;51:218-24.

19. Albuquerque HPC, Vidal PC. Trombose venosa profunda: revisão dos conceitos atuais. Rev Bras Ortop. 1996;31:851-6.

20. Sikorski JM, Hampson WG, Stadsdon GE. The natural history and a etiology of deep vein thrombosis after total hip replacement. J Bone Joint Surg Br. 1981;63-B:171-7.

21. Thomas DP, Meston RE, Wood RD, Hockley DJ. The relationship between vessel wall injury and venous thrombosis: an experimental study. Br J Haematol. 1985;59:449-57.

22. Brenner BM, Troy JL, Ballerman BJ. Endothelium-dependent vascular responses. J Clin Invest. 1989;84:1373-8.

23. Hirsh J, Hull R. Venous thromboembolism: natural history, diagnosis and management. Boca Raton: CRC Press; 1987. p. $17-21$.

24. Green D, Hirsh J, Heit J, Prins M, Davidson B, Lensing AW. Low molecular weight eparin:a critical analysis of clinical trials. Pharmacol Rev. 1994;46:89-10.

25. Merlo I, Parente JB, Komlós PP. Varizes e telangiectasias: diagnóstico e tratamento. Rio de Janeiro: Revinter; 2006.

26. Figueiredo MAM., Filho AD, Cabral ALS. Avaliação do efeito da meia elástica na hemodinâmica venosa dos membros inferiores de pacientes com insuficiência venosa crônica. J Vasc Bras. 2004;3:231-7.

27. Blättler W, Partsch H. Leg compression and ambulation is better than bed rest for the treatment of acute deep venous thrombosis. Int Angiol. 2003;22:393-400.

28. Partsch H. Therapy of deep vein thrombosis with low molecular weight heparin, leg compression and immediate ambulation. Vasa. 2001;30:195-204.

29. Kiser TS, Stefans VA. Pulmonary embolism in rehabilitation patients: relation to time before return to physical therapy after diagnosis of deep vein thrombosis. Arch Phys Me Rehabil. 1997;78:942-5. 
30. Partsch H, Kaulich M, Mayer W. Immediate mobilisation in acute vein thrombosis reduces post-thrombotic syndrome. Int Angiol. 2004;23:206-12.

31. Partsch, H. Ambulation and compression after deep vein thrombosis: dispelling myths. Semin Vasc Surg. 2005;18:148-52.

32. Jünger M, Diehm C, Störiko H, et al. Mobilization versus immobilization in the treatment of acute proximal deep venous thrombosis: a prospective, randomized, open, multicentre trial. Curr Med Res Opin. 2006;22:593-602.

33. Samama MM, Simonneau G, Wainstein J-P, De Vathaire F, Huet Y, Landauer D. SIRIUS Study: Epidemiology of risk factors of deep venous thrombosis of the lower limbs in community practice. Thromb Haemost. 1993;69:797A.

34. Killewich LA, Macko RF, Cox K, et al. Regression of proximal deep venous thrombosis is associated with fibrinolytic enhancement. J Vasc Surg.1997;26:861-8.

35. Fischer H. Eine neue Therapie der Phlebitis. Med Klin. 1910;30:1172-80.

36. Partsch H, Menzinger G, Mostbeck A. Inelastic leg compression is more effective to reduce deep venous refluxes than elastic bandages. Dermatol Surg. 1999;25:695-700.

37. Schulman S. Studies on the medical treatment of deep vein thrombosis. Acta Med Scand Suppl. 1985;704:1-68.
38. Qvarfordt P, Christenson JT, Eköf B, Ohlin P, Saltin B. Intramuscular pressure, muscle blood flow, and skeletal muscle metabolism in chronic anterior tibial compartment syndrome. Clin Orthop Relat Res. 1983;179:284-90.

39. Susan RK, Jeffrey SG. Relationship between deep venous thrombosis and the postthrombotic syndrome. Arch Intern Med. 2004;164:17-26. http://archinte.ama-assn.org/cgi/ content/full/164/1/17?. Acessado: 26/10/2008.

40. Stick C, Grau H, Witzleb E. On the edema: preventing effect of the calf muscle pump. Eur J Appl Physiol. 1989;59:39-47.

41. Padberg FT Jr, Johnson MV, Sisto SA. Structured exercise improves calf muscle pump function in choronic venous insufficiency: a randomized trial. J Vasc Surg. 2004; 39:79-87.

42. Journal of Vascular Surgery [site na Internet]. Elsevier, Inc. http://www.journals.elsevierhealth.com/periodicals/ymva/ article/PIIS0741521403014125/fulltext. Acessado: 12/01/2008.

43. Shrier I, Kahn S R. Effect of physical activity after recent deep venous thrombosis: a cohort study. Med Sci Sports Exerc. 2005;37:630-4.

Correspondência:

Geane de Souza Penha

Rua Coronel Américo, 131, Barreiros

CEP 88117-310 - São José, SC

Tel.: (48) 3240.0040, (48) 9926.1728

E-mail: geanepenha@yahoo.com.br 